\section{Band 19, Heft 3, September 2009}

\section{Editorial}

140 Keine großen Überraschungen, aber interessante Anregungen: Das Forschungsgutachten zur Psychotherapieausbildung ist da Rief, W. (Marburg)

\section{Übersichtsarbeit - Diagnostik}

145 Innovative Ansätze in der klinischen Angstdiagnostik

Helbig-Lang, S.; Petermann, F. (Bremen)

\section{Übersichtsarbeit - Kinder und Jugendliche}

153 Schulabsentismus bei Kindern und Jugendlichen Konzept und Behandlungsempfehlungen Walter, D.; Döpfner, M. (Köln)

Originalarbeiten

161 Sexuelle Dysfunktionen und sexuelle Zufriedenheit bei Patientinnen mit posttraumatischer

Belastungsstörung

Haase, A.; Boos, A.; Schönfeld, S.; Hoyer, J. (Dresden)

169 Dichotomer Antwortstil im Rahmen von achtsamkeitsbasierter kognitiver Therapie Tenbergen, M.; Teismann, T.; Michalak, J. (Bochum)

Kasuistik

177 Traumafokussierte kognitive Verhaltenstherapie mit einem Kriegsopfer: Ein Fallbericht Müller, J. (Zürich)

Interview

185 Jeffrey Young: «Körperkontakt zwischen Therapeut(in) und Patient(in) ist erlaubt» Linden, M. (Berlin)

\section{Vol. 19, Issue 3, September 2009}

Editorial

140 No Big Surprise but Stimulating Suggestions: The Postgraduate Training in Psychotherapy Has Been Scientifically Evaluated Rief, W. (Marburg)

Review Article - Diagnostics

145 Innovative Approaches in Clinical Anxiety Assessment

Helbig-Lang, S.; Petermann, F. (Bremen)

Review Article - Children and Adolescents

153 School Absenteeism in Children and Adolescents

- Concept and Treatment Recommendations

Walter, D.; Döpfner, M. (Köln)

Original Articles

161 Sexual Dysfunctions and Sexual Satisfaction in Female PTSD Patients

Haase, A.; Boos, A.; Schönfeld, S.; Hoyer, J. (Dresden)

169 Dichotomous Response Style and MindfulnessBased Cognitive Therapy

Tenbergen, M.; Teismann, T.; Michalak, J. (Bochum)

Casuistic Contribution

177 Trauma-Focused Cognitive Behavioural Therapy with a Victim of War: A Case Report

Müller, J. (Zürich)

Interview

185 Jeffrey Young: 'Body Contact between Therapist and Patient Is Allowed' Linden, M. (Berlin)

\section{KARGER}

Fax +497614520714

Information@Karger.de

www.karger.com 


\section{Band 19, Heft 3, September 2009}

Pro und Contra

187 Das Psychiatriejahr in der Ausbildung zum Psychologischen Psychotherapeuten Hohagen, F. (Lübeck); Rief, W. (Marburg)

Brief an die Herausgeber

191 Wenn wir über Selbsterfahrung reden, dann müssen wir über Ausbildung reden! Laireiter, A.-R. (Salzburg)

194 Buchbesprechungen

198 Fort- und Weiterbildung

202 Mitteilungen der Verbände

207 Tagungen und Kongresse

210 Hinweise für Autoren

183 Impressum

U2 Erläuterungen zum Titelbild (2. Umschlagseite) Leibl, C.; Weiss, S.; Hauer, M. (Prien am Chiemsee)

Einen Ausblick auf den Inhalt der kommenden Hefte finden Sie auf Seite 212.

\section{Vol. 19, Issue 3, September 2009}

Pro and Con

187 One Year Full-Time Practical Work in Psychiatry in the Clinical Training of Psychological Psychotherapists Hohagen, F. (Lübeck); Rief, W. (Marburg)

Letter to the Editors

191 If We Talk about Self-Awareness, We also Have to Talk about Postgraduate Training Laireiter, A.-R. (Salzburg)

194 Book Reviews

198 Educaton

202 Information by Behavior Therapy Associations

207 Meetings and Conferences

210 Guidelines for Authors

183 Imprint

C2 Explanations on the Title (Inside front cover) Leibl, C.; Weiss, S.; Hauer, M. (Prien am Chiemsee) 